\title{
XMM-Newton first-light observations of the Hickson galaxy group 16
}

M. J. L. Turner ${ }^{1}$, J. N. Reeves ${ }^{1}$, T. J. Ponman ${ }^{2}$, M. Arnaud ${ }^{3}$, M. Barbera ${ }^{16}$, P. J. Bennie ${ }^{1}$, M. Boer $^{4}$, U. Briel ${ }^{5}$, I. Butler ${ }^{2}$, J. Clavel ${ }^{6}$, P. Dhez ${ }^{7}$, F. Cordova $^{8}$, S. Dos Santos ${ }^{1}$, P. Ferrando ${ }^{3}$, S. Ghizzardi $^{9}$, C. V. Goodall ${ }^{2}$, R. G. Griffiths ${ }^{1}$, J. F. Hochedez ${ }^{15}$, A. D. Holland ${ }^{1}$, F. Jansen ${ }^{10}$, E. Kendziorra ${ }^{11}$, A. Lagostina $^{9}$, R. Laine ${ }^{12}$, N. La Palombara ${ }^{9}$, M. Lortholary ${ }^{3}$, K. O. Mason ${ }^{13}$, S. Molendi ${ }^{9}$, C. Pigot $^{3}$, W. Priedhorsky ${ }^{14}$, C. Reppin ${ }^{5}$, R. Rothenflug ${ }^{3}$, P. Salvetat ${ }^{15}$, J. Sauvageot ${ }^{3}$, D. Schmitt ${ }^{3}$, S. Sembay ${ }^{1}$, A. Short ${ }^{1}$, L. Strüder ${ }^{5}$, M. Trifoglio ${ }^{17}$, J. Trümper ${ }^{5}$, S. Vercellone ${ }^{9}$, L. Vigroux ${ }^{3}$, G. Villa ${ }^{9}$, and M. Ward ${ }^{1}$

1 X-ray Astronomy Group, Department of Physics and Astronomy, Leicester University, Leicester LE1 7RH, UK

2 School of Physics and Astronomy, University of Birmingham, B15 2TT, UK

3 CEA Saclay, 91191 Gif-sur-Yvette, France

${ }^{4}$ CESR Toulouse, BP 4346, 31028 Toulouse Cedex 4, France

5 Max-Planck-Institut für extraterrestrische Physik, Postfach 1603, 85748 Garching, Germany

6 ESA-SOC, Vilspa, PO Box 50727, 28080, Madrid, Spain

7 LURE, Bât. 209 D, Université Paris Sud, 91405 Orsay, France

8 Office of Research, University of California, Santa Barbara, CA 93106, USA

9 IFC Milan, 20133 Milano, Italy

10 PS Estec, Postbus 299, 2200 AG Noordwijk, The Netherlands

11 IAAP Tuebingen, 72076, Germany

12 PX Estec, Postbus 299, 2200 AG Noordwijk, The Netherlands

13 MSSL Holmbury St Mary, Dorking RH5 6NT, UK

14 LANL, SST9, MS D436, Los Alamos, NM 87545, USA

15 Institut d'Astrophysique Spatiale, Bât. 121, Université Paris Sud, 91405 Orsay, France

16 Osservatorio Astronomico di Palermo, Palermo 90134, Italy

17 ITESRE, 41010 Bologna, Italy

Received 13 October 2000 / Accepted 27 October 2000

\begin{abstract}
This paper presents the $X M M$-Newton first-light observations of the Hickson-16 compact group of galaxies. Groups are possibly the oldest large-scale structures in the Universe, pre-dating clusters of galaxies, and are highly evolved. This group of small galaxies, at a redshift of 0.0132 (or $80 \mathrm{Mpc}$ ) is exceptional in the having the highest concentration of starburst or AGN activity in the nearby Universe. So it is a veritable laboratory for the study of the relationship between galaxy interactions and nuclear activity. Previous optical emission line studies indicated a strong ionising continuum in the galaxies, but its origin, whether from starbursts, or AGN, was unclear. Combined imaging and spectroscopy with the EPIC X-ray CCDs unequivocally reveals a heavily obscured AGN and a separately identified thermal (starburst) plasma, in NGC 835, NGC 833, \& NGC 839. NGC 838 shows only starburst thermal emission. Starbursts and AGN can evidently coexist in members of this highly evolved system of merged and merging galaxies, implying a high probability for the formation of AGN as well as starbursts in post-merger galaxies.
\end{abstract}

Key words. galaxies: active - galaxies: starburst $-\mathrm{X}$-rays: galaxies

\section{Introduction}

The Hickson-16 galaxy group (or HCG-16) comprises seven galaxies with a mean recession velocity (Ribeiro et al. 1996) of $3959 \pm 66 \mathrm{~km} \mathrm{~s}^{-1}$ and a velocity dispersion of $86 \pm 55 \mathrm{~km} \mathrm{~s}^{-1}$, centered on the position $\alpha=02^{\circ} 09^{\prime} 33^{\prime \prime}$, $\delta=-10^{\circ} 09^{\prime} 46.7^{\prime \prime}(\mathrm{J} 2000)$. The four central members of the group, originally identified by Hickson (Hickson 1982), all fall within the $30^{\prime}$ field of view of EPIC, they all show evidence for mergers and a strong ionising continuum; they have well resolved optical nuclei (Mendes de Oliveira et al. 1998). However the nature of the ionising continuum 


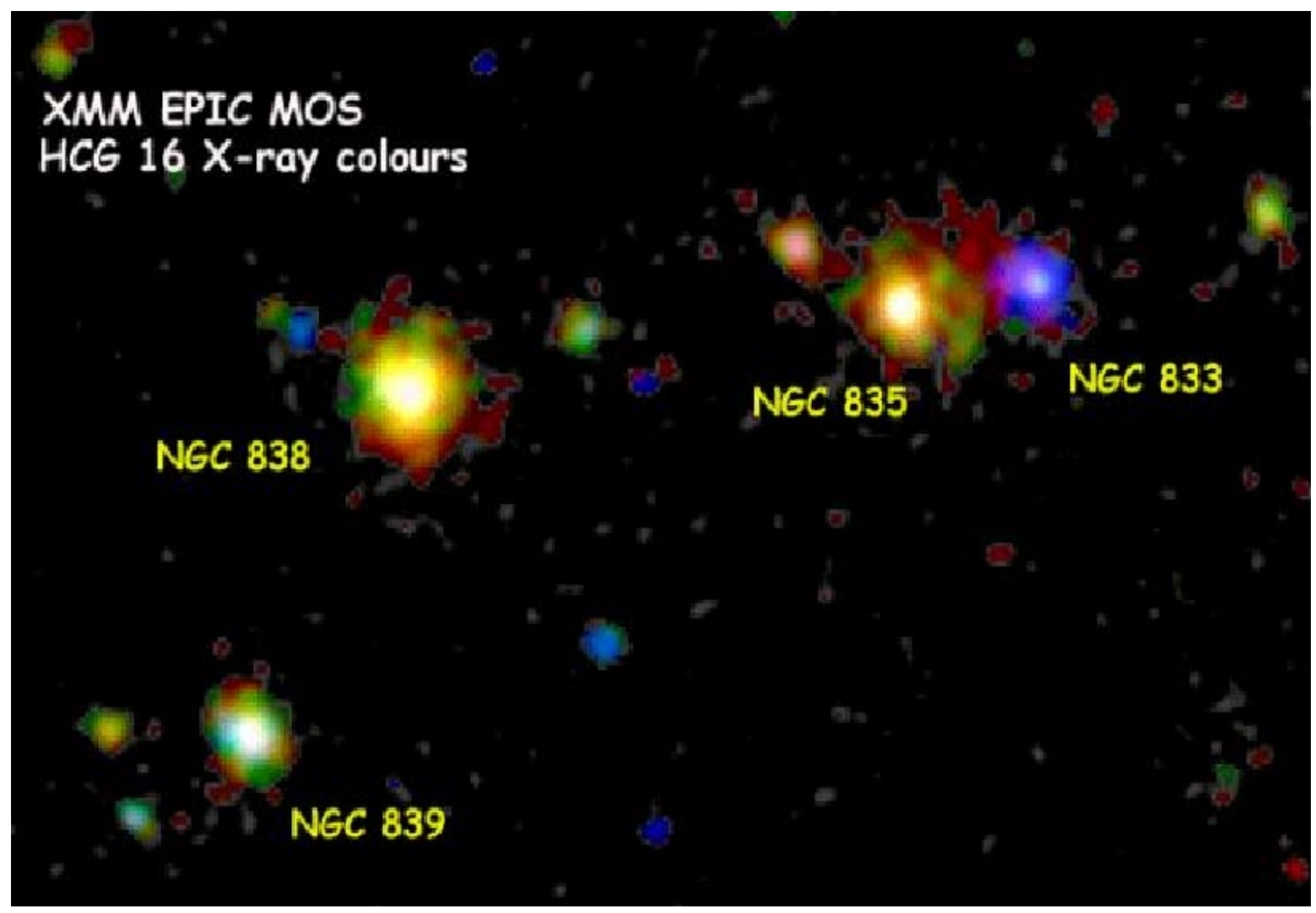

Fig. 1. The smoothed colour X-ray image of HGC 16 made with the EPIC MOS CCD imaging spectrometers on $X M M$ Newton. The spatial resolution is $6^{\prime \prime}$ Full Width at Half Maximum, and $15^{\prime \prime}$ Half Energy Width, limited by the mirrors. The energy band is $0.2-10 \mathrm{keV}$ and the energy resolution varies from $140 \mathrm{eV}$ FWHM at $6 \mathrm{keV}$ to $70 \mathrm{eV}$ at $500 \mathrm{eV}$. In the colour image, red corresponds to $800 \mathrm{eV}$ and blue to $>3 \mathrm{keV}$. The physical scale across the image corresponds to $200 \mathrm{kpc}$ (using $H_{0}=50 \mathrm{~km} \mathrm{~s}^{-1} \mathrm{Mpc}^{-1}$ ). Notice the very hard (blue) nucleus of NGC 833 and the soft (red) halo emission around the companion galaxy NGC 835

is unclear. Optical emission line diagnostics (de Carvalho \& Coziol 1999; Veilleux \& Osterbrock 1987) suggest that in NGC 838 and NGC 835 there is a starburst nucleus, whilst in NGC 835, NGC 833 and NGC 839 there is a low ionisation narrow emission line region (or LINER 2 nucleus). This could arise either from an AGN or from starburst activity: $[\mathrm{OIII}] / \mathrm{H} \beta$ values for the nuclei are all $<2.5$ (de Carvalho \& Coziol 1999). The EPIC instrument is able to observe directly the ionising continuum, and distinguish clearly between optically thin thermal emission from a starburst, and non-thermal hard X-ray emission from an AGN. These observations can therefore be used to determine the nature of the ionising continuum, helping to clarify the relationship between mergers, the triggering of starbursts, and the creation and fueling of black holes.

\section{The XMM-Newton observations}

The $X M M$-Newton Observatory (Jansen et al. 2001) has three X-ray telescopes of area $\sim 1500 \mathrm{~cm}^{2}$, with the three EPIC instruments at the foci; two of the EPIC imaging spectrometers use MOS CCDs (Turner et al. 2001; Holland et al. 1996) and one uses a PN CCD (Strüder et al. 2001). The observations of the HCG-16 galaxy group were taken in orbit-23 as part of the $X M M$-Newton EPIC first-light. Exposures of $50 \mathrm{ksec}$ were taken with EPIC (sensitive from 0.2 to $10 \mathrm{keV}$ ) and $1 \mathrm{ksec}$ exposures were taken in $V(550 \mathrm{~nm})$ and $U V(280 \mathrm{~nm})$ with the $X M M-N e w t o n$ Optical/UV Monitor (OM) telescope (Mason et al. 2001).

The EPIC data were processed using the pipeline scripts EMCHAIN (MOS) and EPCHAIN (PN). Screening was applied using the XMM-Newton SAS (Science Analysis Software). Hot and bad pixels and negative E3 events were removed from the data to reduce the level of electronic noise. A low energy cut of $200 \mathrm{eV}$ was applied to the data. The first $10 \mathrm{ksec}$ of data were also removed from the EPIC observation, as this contained a high count-rate background particle flare. The resultant exposure time for each of the detectors was $\sim 40 \mathrm{ksec}$.

Figure 1 shows the resultant EPIC X-ray colour image of the centre of the HCG-16 field. The hard, absorbed, 


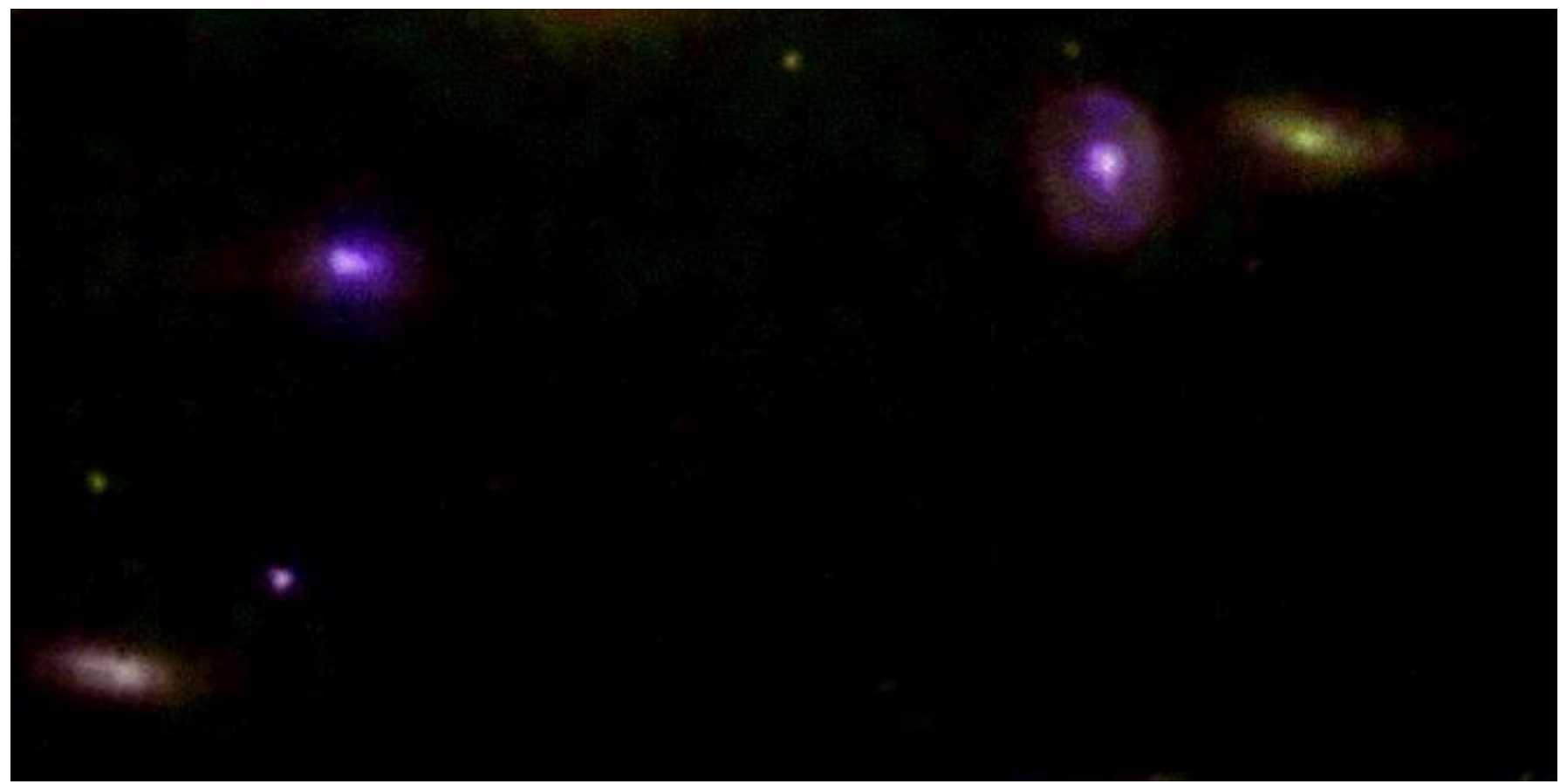

Fig. 2. The OM colour image of HCG 16 derived from 1000 s exposures in $V$ and $U V$ bands. A bright foreground star has been removed from the lower right of the image. Blue here represents the $U V$ band

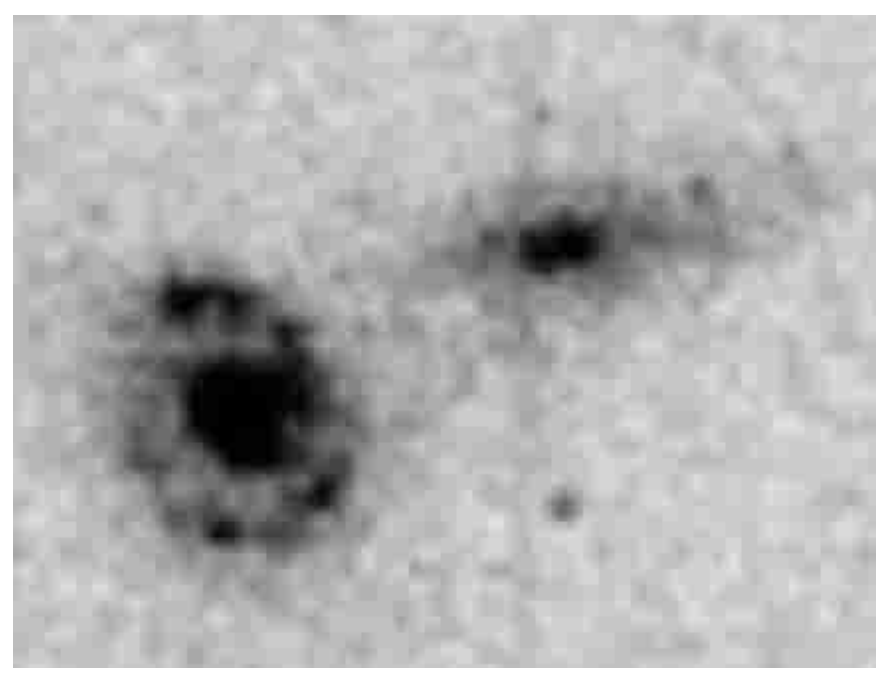

Fig. 3. A close-up, greyscale $U V$ image from the Optical Monitor, showing the galaxies NGC 833 (right) and NGC 835 (left). Bright $U V$ knots, corresponding to possible regions of star formation, are seen in the outer disk of NGC 835

spectrum of the AGN in NGC 833 shows up as a blue point source, and the soft starburst emission in the outer regions of NGC 835 shows as a red halo; the other galaxies show extended X-ray discs. Figure 2 also shows the $V-U V$ colour image from the OM. The nuclear regions of NGC 835 and NGC 838 show up brightly in the ultraviolet, indicative of hot stars or gas associated with enhanced star formation. There are also bright $U V$ knots in the outer regions of NGC 835 showing enhanced star formation there. A close-up OM image of NGC 835 is shown in Fig. 3.

\section{Spectral analysis of the HCG-16 galaxies}

Since EPIC resolves the optical disks of the galaxies, the spectra were prepared from photons falling within a region of interest based on the X-ray image. For NGC 833 the X-ray source is point-like, while for NGC 835 the core and the surrounding region (the red halo in Fig. 1) were analysed separately, the spectra of the remaining galaxies were made using the entire X-ray disc. Background spectra were taken from source-free regions on the central EPIC-MOS and PN chips; the background spectra were normalised to the area of the source extraction regions.

The background subtracted EPIC spectra were fitted, using XSPEC V11.0, with the latest response matrices produced by the EPIC team; the systematic level of uncertainty is $<5 \%$. Finally spectra were binned to a minimum of 20 counts per bin, in order to apply the $\chi^{2}$ minimisation technique. All subsequent errors are quoted to $90 \%$ confidence $\left(\Delta \chi^{2}=4.6\right.$ for 2 interesting parameters $)$. Values of $H_{0}=50 \mathrm{~km} \mathrm{~s}^{-1} \mathrm{Mpc}^{-1}$ and $q_{0}=0.5$ have been assumed and all fit parameters are given in the rest-frame of the HCG-16 system. We now present the individual EPIC spectra of the 4 main Hickson-16 galaxies.

\subsection{NGC 833}

Optical imaging data on NGC 833 reveal a disturbed velocity field and pronounced misalignment of the kinematic and stellar axes, indicative of an ongoing interaction (Mendes de Oliveira et al. 1998). The emission lines present in the optical spectra (de Carvalho \& Coziol 1999) indicate weak non-stellar LINER-2 activity in the core; there is no optical evidence for current star formation 


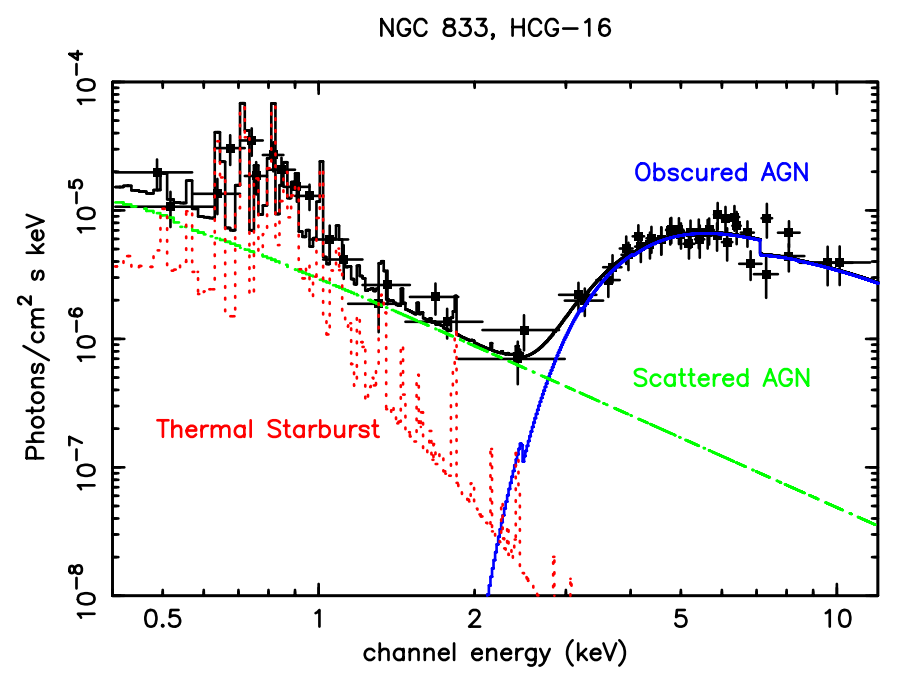

Fig. 4. The X-ray spectrum of the galaxy NGC 833. The most striking feature is the high-energy, absorbed power-law (at $>3 \mathrm{keV}$ ) that is the direct emission from the active black hole at the centre of the galaxy. There is also an un-absorbed power-law, resulting from radiation scattered into our line of sight, by material directly illuminated by the AGN. These two spectral components together show the presence of an AGN of luminosity $1.4 \pm 0.610^{42} \mathrm{erg} \mathrm{s}^{-1}$. There is also weak soft X-ray emission from an optically thin plasma, perhaps originating from starburst activity

([NII] $/ \mathrm{H} \beta \sim$ unity). The EPIC image of this galaxy is point-like, much smaller than the stellar disc.

The best-fitting EPIC X-ray spectrum (Fig. 4) shows three distinct components, all required at $>99.99 \%$ confidence. The most obvious is the peak at high energies from an obscured AGN; this emission is in the form of a power-law of index $\Gamma=1.8 \pm 0.5$, absorbed by material of column density equal to $N_{\mathrm{H}}=2.4 \pm 0.410^{23} \mathrm{~cm}^{-2}$. The second component is an un-absorbed power-law, resulting from radiation scattered into our line of sight, by thin, hot, plasma directly illuminated by the AGN. The third component is radiation from an optically-thin thermal plasma, with a temperature of $k T=470 \mathrm{eV}$. The improvement in the fit upon adding the thermal emission is $\Delta \chi^{2}=36.7$. A summary of the fits to NGC 833 (and the other 3 galaxies) are given in Table 1 .

This complex X-ray spectrum amply confirms the presence of an AGN in NGC 833 of luminosity $1.4 \pm$ $0.610^{42} \mathrm{erg} \mathrm{s}^{-1}$, it is, remarkably, the dominant source of power in the galaxy. In contrast, the thermal X-ray emission, is more than 100 times weaker $\left(8.9 \pm 3.010^{39} \mathrm{erg} \mathrm{s}^{-1}\right)$ and the FIR luminosity (Verdes-Montenegro et al. 1998) is also very low $\left(<310^{42} \operatorname{erg~s}^{-1}\right)$.

\subsection{NGC 835}

The adjacent galaxy, NGC 835 is undergoing a gravitational interaction with its neighbour NGC 833, as evidenced by the tidal tails in the optical image; and apparently contiguous stellar discs (Mendes de Oliveira et al. 1998). The velocity field is normal, but there is emission
NGC 835, HCG-16

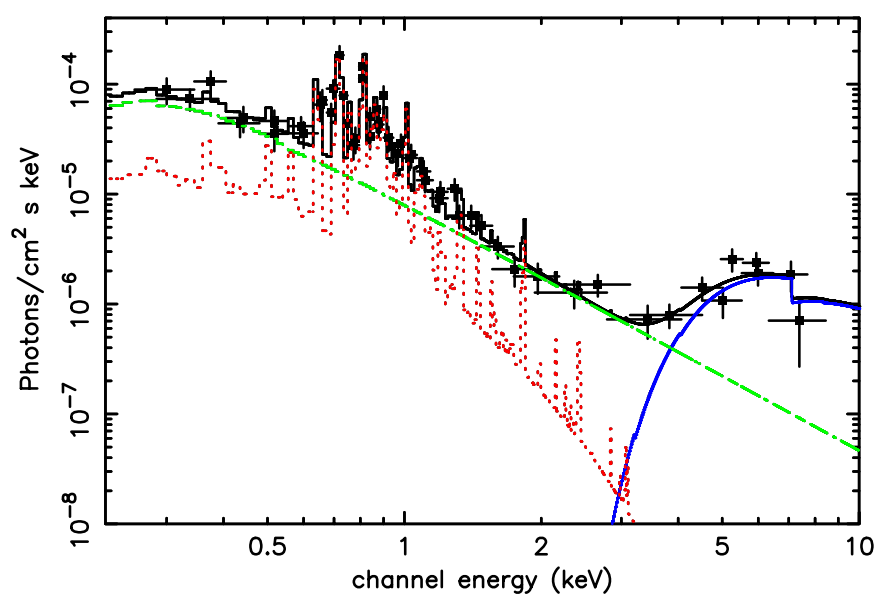

Fig. 5. The spectrum of the companion galaxy, NGC 835, which also shows an obscured active nucleus together with strong thermal soft X-ray emission. The AGN in both NGC 833 and NGC 835 may have been triggered by mutual gravitational interaction

line evidence (de Carvalho \& Coziol 1999) for LINER nuclear activity, and for current starburst activity in the outer regions; the knotted ring structure seen in the OM image supports this.

The X-ray emission from NGC 835 can be spatially separated into two areas, the core, and an outer region corresponding to the remainder of the stellar disc. The core has a very similar spectrum (Fig. 5) to that of NGC 833. There are absorbed and scattered power-laws indicating a heavily obscured AGN $\left(N_{\mathrm{H}}=4.6 \pm 1.510^{23} \mathrm{~cm}^{-2}\right)$ of luminosity $1.210^{42} \mathrm{erg} \mathrm{s}^{-1}(0.5-10 \mathrm{keV})$, but the soft thermal component is more luminous than NGC 833 at $2.5 \pm$ $0.310^{40} \mathrm{erg} \mathrm{s}^{-1}$; it is almost certainly from current starburst activity and the FIR luminosity (Verdes-Montenegro et al. 1998) is 100 times larger at $2.710^{44} \mathrm{erg} \mathrm{s}^{-1}$. The spectrum at the periphery of NGC 835 is purely thermal, with a temperature of $300 \mathrm{eV}$ and a luminosity of $2.9 \pm 0.710^{40} \mathrm{erg} \mathrm{s}^{-1}$, similar to that of the core. This is the X-ray emission from the starburst region including the ring structure and knots seen in the OM $V-U V$ image.

To summarize, the EPIC data clearly show the presence of an AGN, in both NGC 833 and NGC 835, that coexists with present starburst activity in the core (and for NGC 835 in the periphery) of the galaxies. The detections of the obscured AGN and thermal starburst components in both galaxies are highly significant, at $>99.99 \%$ confidence (see Table 1).

\subsection{NGC 838}

NGC 838 is an ongoing merger with strong starburst activity. Optical data (Mendes de Oliveira et al. 1998) show kinematic warping, and multiple velocity components in the ionised gas, and a double optical core (also see de Carvalho \& Coziol 1999). The infrared luminosity 
Table 1. X-ray spectral fits to the 4 HCG-16 galaxies. ${ }^{a}$ Temperature of thermal component in keV. ${ }^{b}$ Column density of the absorbed power-law in units of $10^{22} \mathrm{~cm}^{-2}$. ${ }^{c}$ Improvement in the spectral fit upon adding the obscured hard power-law. ${ }^{d}$ Improvement in the fit from adding a soft, thermal (Mekal) component. ${ }^{e}$ Best-fit reduced chi-squared. ${ }^{f}$ Indicates that parameter is fixed. Note ABS.PL is the absorbed power-law, SCAT.PL is the scattered power-law; $\Gamma$ for these 2 components have been tied

\begin{tabular}{|c|c|c|c|c|c|c|c|}
\hline \multirow[b]{2}{*}{ Galaxy } & \multirow[b]{2}{*}{ Model } & \multicolumn{2}{|c|}{ Hard Component } & \multicolumn{4}{|c|}{ Thermal Soft Component } \\
\hline & & $\Gamma$ or $k T^{a}$ & $N_{\mathrm{H}}^{b}$ & $\Delta \chi^{2 c}$ & $k T^{a}$ & $\Delta \chi^{2 d}$ & $\chi_{\nu}^{2 e}$ \\
\hline NGC 833 & ABS.PL + SCAT.PL + MEKAL & $\Gamma=1.8 \pm 0.5$ & $24 \pm 4$ & 92.7 & $0.47 \pm 0.12$ & 36.7 & 0.632 \\
\hline NGC 835 (centre) & ABS.PL + SCAT.PL + MEKAL & $\Gamma=2.25 \pm 0.23$ & $46 \pm 15$ & 58.4 & $0.51 \pm 0.07$ & 176.2 & 1.02 \\
\hline NGC 835 (diffuse) & MEKAL $\times 2$ & $k T=4 \mathrm{keV}^{f}$ & - & - & $0.31 \pm 0.05$ & 73.4 & 1.2 \\
\hline NGC 838 & MEKAL $\times 2$ & $k T=3.2 \pm 0.8$ & - & - & $0.59 \pm 0.04$ & 209.5 & 1.21 \\
\hline NGC 839 & ABS.PL + SCAT.PL + MEKAL & $\Gamma=2.1 \pm 0.8$ & $45 \pm 20$ & 12.0 & $0.63 \pm 0.10$ & 40.9 & 1.38 \\
\hline
\end{tabular}

NGC 838, HCG-16

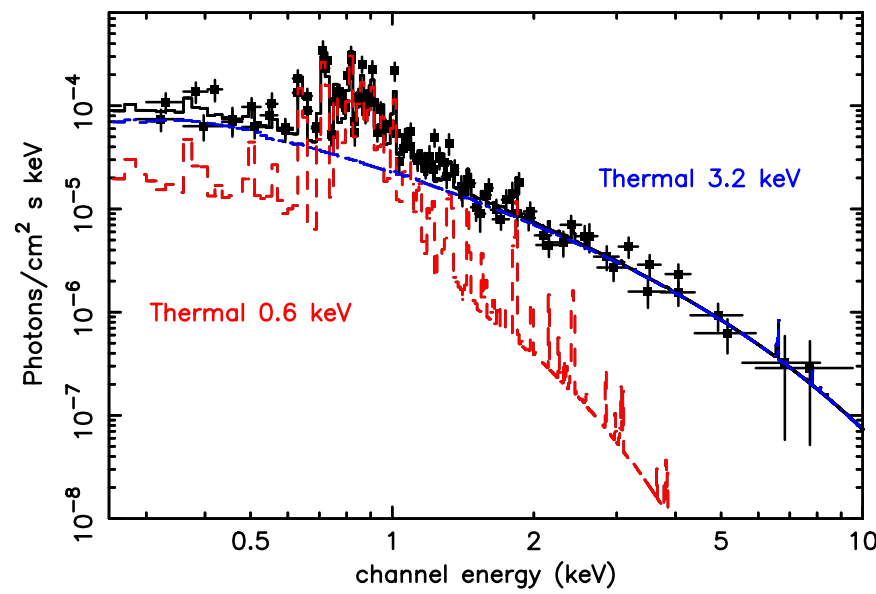

Fig. 6. The EPIC-MOS spectrum of NGC 838. Only emission from the starburst is present, with no detectable hard X-ray emission from a central AGN; the hard X-ray emission could arise from unresolved X-ray binaries in the galaxy

is $3.3 \pm 10^{44} \mathrm{erg} \mathrm{s}^{-1}$. The EPIC spectrum of NGC 838 (Fig. 6) shows purely thermal emission, the disc is resolved in X-rays, but there is no separate sharp core in the $\mathrm{X}$-ray image. The spectrum is fitted with a two temperature thermal spectrum $(k T=3.2 \mathrm{keV}$ and $k T=590 \mathrm{eV})$ and the luminosity is high at $1.9 \pm 0.310^{41} \mathrm{erg} \mathrm{s}^{-1}$. This is all consistent with the optical data: the soft X-ray emission is from the ionised gas produced in the starburst while the hard thermal spectrum could be characteristic of unresolved X-ray binaries. There is no statistically significant scattered or obscured power law; the upper limit for the AGN luminosity is $510^{40} \mathrm{erg} \mathrm{s}^{-1}$, assuming an absorbing column of $510^{23} \mathrm{~cm}^{-2}$.

\subsection{NGC 839}

NGC 839 may also be a recent merger (Mendes de Oliveira et al. 1998; de Carvalho \& Coziol 1999), it has a double nucleus in the optical, a FIR luminosity of $3.110^{44} \mathrm{erg} \mathrm{s}^{-1}$, and a disturbed velocity field; optical lines indicate an active LINER-2 nucleus (de Carvalho \& Coziol 1999). In the soft X-ray EPIC spectrum (Fig. 7) there is optically-thin thermal emission, similar to that of the other galaxies,

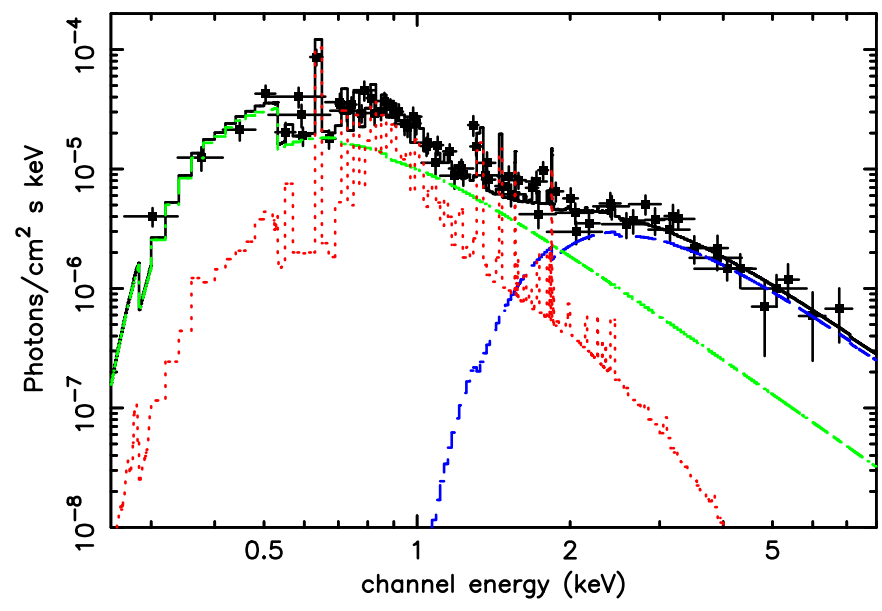

Fig. 7. The X-ray spectrum of NGC 839. There is both starburst emission and emission from a low luminosity obscured AGN. Unusually the elemental abundance of NGC 839 appears to be $\sim 5$ times solar

of temperature $k T=600 \mathrm{eV}$, and luminosity $1.8 \pm$ $0.310^{40} \mathrm{erg} \mathrm{s}^{-1}$; a typical indicator of a current starburst. The spectrum also shows an obscured AGN, as found in NGC 835 \& NGC 833; it is however much less luminous $\left(8 \pm 310^{40} \mathrm{erg} \mathrm{s}^{-1}\right)$ for a column of $N_{\mathrm{H}}=510^{23} \mathrm{~cm}^{-2}$. Interestingly the abundances in NGC 839 appear to be higher than solar. There are apparent weak Lyman- $\alpha$ lines of $\mathrm{O}, \mathrm{Mg}$ and $\mathrm{Si}$ in the EPIC spectrum, although the significance of these features is low (at only $90 \%$ confidence). Fitting the soft X-ray spectrum with the MEKAL model does however yield an over-abundance of $5.2 \pm 2.0$ times the solar value. One interesting possibility is that the heavier elements have been enriched through the intense starburst activity in this galaxy.

\section{Conclusions}

Direct X-ray spectroscopy is the best way to identify hidden AGN in galaxies, and here the EPIC cameras on $X M M-N e w t o n$ have produced clear evidence for active, massive black holes in three out of four galaxies in HCG16. The presence of a similar active nucleus in NGC 838 is unlikely, unless it is very heavily absorbed. The nature 
of the ionising continuum in the four galaxies has been elucidated: there is thermal emission from starburst activity in three, (possibly four) of the galaxies, and in three of them there is a coexisting active black hole. While LINER-1 galaxies with broad $\mathrm{H} \beta$ lines do harbour black holes (Terashima et al. 1998, 2000) this is the first direct evidence that black holes power LINER2 galaxies. These AGN are at the low end of the luminosity scale, consistent with their small size (Magorrian et al. 1998).

In NGC 833, the accreting black hole X-ray luminosity arguably exceeds the FIR luminosity. This is very unusual, even compared with much more luminous AGN, and may indicate the stripping of dust and gas by past interactions with other galaxies (Mendes de Oliveira et al. 1998). The EPIC X-ray study of this nearby and evolved system of small galaxies indicates a high fraction of active black holes coexisting with starbursts. This is consistent with optical studies of compact groups (e.g. Coziol et al. 2000), where a large fraction of galaxies with nuclear activity is found. The observations may also provide a link between normal galaxies, where black holes may be inactive, and Seyfert galaxies and quasars, where black holes dominate, and where galaxy mergers may be implicated in the onset of black hole activity (Boyce et al. 1996; Bahcall et al. 1997). Further observations with $X M M$-Newton have the potential to determine more precisely the fraction of nearby galaxies that harbour active, low-luminosity black holes.

Acknowledgements. This work is based on observations obtained with $X M M-N e w t o n$, an ESA science mission with instruments and contributions directly funded by ESA Member States and the USA (NASA). EPIC was developed by the EPIC Consortium led by the Principal Investigator, Dr. M. J. L. Turner. The consortium comprises the following Institutes: University of Leicester, University of Birmingham, (UK); CEA/Saclay, IAS Orsay, CESR Toulouse, (France); IAAP Tuebingen, MPE Garching, (Germany); IFC Milan, ITESRE Bologna, OAPA Palermo, (Italy). EPIC is funded by: PPARC, CEA, CNES, DLR and ASI. Finally we thank the referee, Reinaldo de Carvalho, for his report, and for some useful comments and suggestions.

\section{References}

Bahcall, J. N., Kirhakos, S., \& Saxe, D. 1997, ApJ, 479, 642

Boyce, P. J., Disney, M. J., Blades, J. C., et al. 1996, ApJ, 473, 760

Coziol, R., Iovino, A., \& de Carvalho, R. R. 2000, AJ, 120, 47

de Carvalho, R. R., \& Coziol, R. 1999, AJ, 117, 1657

Hickson, P. 1982, ApJ, 255, 382

Holland, A. D., Turner, M. J. L., Abbey, A. F., \& Pool, P. 1996, SPIE, 2808414

Jansen, F., Lumb, D., Altieri, B., et al. 2001, A\&A, 365, L1

Magorrian, J., Tremaine, S., Richstone, D., et al. 1998, AJ, 115,2285

Mason, K. O., Breeveld, A., Much, R., et al. 2001, A\&A, 365, L36

Mendes de Oliveira, C., Plana, H., Amram, P., Bolte, M., \& Boulesteix, J. 1998, ApJ, 507, 691

Ribeiro, A. L. B., de Carvalho, R. R., Coziol, R., Capelato, H. V., \& Zepf, S. E. 1996, ApJ, 463, L5

Strüder, L., Briel, U., Dennerl, K., et al. 2001, A\&A, 365, L18

Terashima, Y., Kunieda, H., Misaki, K., et al. 1998, ApJ, 503, 312

Terashima, Y., Ho, L. C., Ptak, A. F., \& Kunieda, H. 2000, ApJ, 533, 729

Turner, M. J. L., Abbey, A., Arnaud, M., et al. 2001, A\&A, 365, L27

Veilleux, S., \& Osterbrock, D. E. 1987, ApJS, 63, 295

Verdes-Montenegro, L., Yun, M. S., Perea, J., del Olmo, A., \& Ho, P. T. B. 1998, ApJ, 497, 89 